\title{
Alterações biomecânicas durante o período gestacional: uma revisão
}

\author{
Luana Mann ${ }^{1}$ \\ Julio Francisco Kleinpaul ${ }^{2}$ \\ Carlos Bolli Mota ${ }^{3}$ \\ Saray Giovana dos Santos ${ }^{4}$ \\ ${ }^{1}$ Mestranda em Educação Física, Laboratório de Biomecânica, Centro de Desportos da \\ Universidade Federal de Santa Catarina, Florianópolis, SC, Brasil \\ ${ }^{2}$ Mestre em Educação Física, Laboratório de Biomecânica, Centro de Desportos da \\ Universidade Federal de Santa Catarina, Florianópolis, SC, Brasil \\ ${ }^{3}$ Professor Doutor em Ciência do Movimento Humano, Laboratório de Biomecânica, Centro \\ de Educação Física e Desportos da Universidade Federal de Santa Maria, RS, Brasil \\ ${ }^{4}$ Professora Doutora em Engenharia de Produção, Laboratório de Biomecânica, Centro de \\ Desportos da Universidade Federal de Santa Catarina, Florianópolis, SC, Brasil
}

\begin{abstract}
Resumo: O objetivo dessa revisão foi fazer uma análise dos estudos que investigaram o comportamento das variáveis cinéticas e cinemáticas da marcha humana e do controle da postura corporal durante a gestação, bem como verificar a ocorrência de quedas. Para isso, foi feita uma busca por referências em língua Portuguesa e Inglesa, utilizando-se bases eletrônicas de dados: SciELO, Sciencedirect, Scopus, Pubmed Central e Pubmed. Foram considerados artigos originais que tratassem de forma clara e objetiva 0 assunto, e publicados entre 1990 e 2009 (julho). De modo geral mudanças significativas são observadas nas variáveis cinéticas e cinemáticas da marcha e do equilíbrio a partir do terceiro trimestre da gestação, nesse período a incidência de quedas também é maior. Muitos são os fatores relacionados a estas mudanças, mas o aumento da massa corporal é o principal destes.
\end{abstract}

Palavras-chave: Gestação. Equilíbrio. Marcha. Quedas.

\section{Biomechanical changes during pregnancy: a review}

\begin{abstract}
The purpose of this review was to analyze the studies that investigated the behavior of the kinetics and kinematics of human gait and posture control during pregnancy, and to verify the occurrence of falls. For this, a search was made for references in Portuguese and English, using search engines: PubMed, Sciencedirect, Scopus, and Pubmed Pubmed Central. Original papers published from 1990 to 2009 (july) were considered when the main subject of discussion was the topic of interest. Overall significant changes are observed on the kinetic and kinematic variables of gait and balance from the third trimester of pregnancy during this period the incidence of falls is also larger. There are many factors related to these changes, but the increase in body mass is the most important of these.
\end{abstract}

Key Words: Pregancy. Balance. Gait. Falls.

\section{Introdução}

A gravidez consiste de um processo fisiológico natural compreendido pela seqüência de adaptações ocorridas no corpo da mulher a partir da fertilização. A preparação do corpo para a gestação envolve ajustes dos mais variados sistemas (MANTLE e POLDEN, 2005) e pode ser considerada um estado de saúde que envolve mudanças fisiológicas iguais ou maiores do que as que acompanham muitos estados patológicos (SANTOS, 1998). As mudanças na mecânica do esqueleto são devido a ação hormonal que aumenta a frouxidão ligamentar (MARNACH et $\underline{\text { al., 2003) e mudanças biomecânicas (BIRCH et }}$ al., 2003) que provocam modificações estruturais na estática e dinâmica do esqueleto.

A marcha é uma atividade simples da vida diária e uma das principais habilidades do ser humano. Constitui-se em um dos mais complexos e integrados movimentos realizados pelo ser humano (WINTER, 1995). A marcha está entre os atos motores mais automatizados, e a seqüência de eventos que geram o andar é altamente repetitiva de ciclo após ciclo e também entre diferentes indivíduos. Tal regularidade permite que se estabeleçam critérios objetivos para a distinção entre padrões normais e patológicos, bem como para a discriminação daquelas mudanças qualitativas causadas pelo 
desenvolvimento do indivíduo (MANN et al., 2008b). Mesmo constituindo-se de um gesto natural acredita-se que padrões diferenciados aconteçam durante sua realização com 0 processo de gestação. Estes podem acarretar dores e desconfortos durante outras atividades da vida diária e profissional (MANN et al., 2009).

Assim como a marcha, um bom equilíbrio é indispensável ao ser humano, pois está presente nas atividades da vida diária, sendo seu controle e correto funcionamento atribuído aos sistemas sensorial, motor e sistema nervoso central (HORAK e MACPHERSON, 1996). Ajustes no sistema postural podem ser esperados com a gestação, advindos do crescimento do útero (anteriorizado dentro da cavidade abdominal), além do aumento no peso e no tamanho das mamas, que são fatores que contribuem para 0 deslocamento do centro de gravidade da mulher para cima e para frente, podendo acentuar a lordose lombar e promover uma anteversão pélvica e mudança na base de apoio (CONTI, CALDERON e RUDGE, 2003). Estas alterações podem acarretar além de dores e desconfortos, quedas que são responsáveis por sérios problemas de saúde que representam 17-39\% dos traumas maternos (DUNNING et al., 2003) e respondem por $3-7 \%$ das mortes fetais (CONNOLLY et al., 1997; WEISS et al., 2001).

Alguns estudos foram realizados a fim de investigar o comportamento da marcha (JENSEN, DOUCET e TREITZ, 1996; PAUL, SALK e FRINGS-DRESEN, 1996; FRANKLIN e CONNER-KERR, 1998; CARPES et al., 2008) e do controle da postura (FOTI, BAGKY e DAVID, 1998; SANTOS, 1998; FOTI et al., 2000; GILLEARD, CROSBIE e SMITH, 2002) porém estudos que descrevam quais são as maiores mudanças advindas do desenvolvimento da gestação ainda são escassos e controversos. Dessa forma, considerando a relevância do tema e o crescente interesse nessa área, definiu-se para este estudo a seguinte situação problema: qual é a incidência de quedas e qual é o comportamento das variáveis cinéticas $e$ cinemáticas da marcha humana e do controle da postura corporal durante a gestação?

Para poder responder a questão problema, este estudo objetivou fazer uma análise dos estudos que investigaram o comportamento das variáveis cinéticas e cinemáticas da marcha humana e do controle da postura corporal durante a gestação, bem como verificar a ocorrência de quedas.

Justifica-se a realização deste estudo tendo em vista a necessidade de se conhecer os ajustes que ocorrem na marcha e no controle postural durante a gestação, a fim de minimizar desconfortos e dores, e evitar possíveis prejuízos como morte fetal.

\section{Métodos}

Para o desenvolvimento do presente estudo foram considerados artigos originais, artigos de revisão, dissertações de mestrado e livros com menção ao tema proposto, que tratassem de forma clara e objetiva o assunto. Realizou-se uma revisão, em estudos indexados no período de 1990 a 2009, utilizando cinco bases eletrônicas de dados, sendo, Scopus $\AA$, SciELO $\AA$, ScienceDirect $\AA^{\circledR}$ (Elsevier), PubMed $\AA$ e PubMed

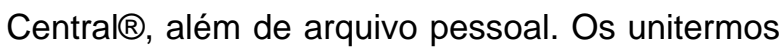
empregados para a busca dos artigos, de acordo com os descritores em ciências da saúde (DeCS), foram: equilíbrio corporal e gestação; quedas e gestação; marcha e gestação e suas respectivas traduções para a língua inglesa. Recorreu-se aos operadores lógicos "AND" e "OR" para combinação dos descritores e termos utilizados para rastreamento das publicações.

A busca nas bases eletrônicas de dados foi realizada no mês de julho de 2009. Após a primeira análise, com avaliação dos títulos, 32 artigos foram considerados elegíveis para a segunda fase desta revisão, que consistiu da leitura dos resumos. Após avaliação dos resumos, os estudos que preenchiam os critérios de inclusão foram lidos na íntegra. Ao final, 19 estudos atenderam a todos os critérios de inclusão, além de 18 estudos com considerações sobre os assuntos.

Para definir quais desses fariam parte da revisão, adotaram-se alguns critérios:

1) o estudo ser original e publicado na língua portuguesa ou inglesa;

2) ter sido publicado no período de 1990 a 2009;

3) citar em seu título ou em seu resumo algum dos descritores.

Foram excluídos os estudos:

1) de validação;

2) resumos em eventos.

Os resultados dos estudos que satisfizeram os critérios adotados estão contidos no quadro 1. 
Quadro 1. Estudos, listados em ordem cronológica considerando os autores, o tamanho da amostra (n), tempo de gestação, a idade, o que o estudo avaliou e o instrumento utilizado para a coleta dos dados.

\begin{tabular}{|c|c|c|c|c|c|}
\hline Autor & $\mathbf{n}$ & Tempo de gestação & Idade (anos) & Avaliou & Instrumento de medida \\
\hline$\frac{\text { Jensen, Doucet e Treitz }}{\underline{(1996)}}$ & 15 & $2^{\circ}$ e $3^{\circ} \operatorname{tr}$ & $26,9 \pm 5,6$ & Controle postural & $\begin{array}{c}\text { Sistema de análise postural } \\
\text { estática }\end{array}$ \\
\hline$\frac{\text { Paul, Salk e Frings-Dresen }}{(1996)}$ & 16 & $10 s ; 20 s ; 30 s ; 40 s$ & $30,4 \pm 2,9$ & Controle postural & $\begin{array}{c}\text { Sistema de análise postural } \\
\text { estática }\end{array}$ \\
\hline$\frac{\text { Franklin e Conner-Kerr }}{(1998)}$ & $12 \mathrm{G}$ & $\begin{array}{l}1^{\circ} \mathrm{tr} \\
3^{\circ} \mathrm{tr}\end{array}$ & 20 a 40 & Controle postural & Antropometria \\
\hline Foti, Bagky e David (1998) & 10 & $\begin{array}{l}3^{\circ} \mathrm{tr} \\
1 \text { ano } \mathrm{pp}\end{array}$ & NR & $\begin{array}{c}\text { Cinética e } \\
\text { cinemática marcha }\end{array}$ & $\begin{array}{c}\text { Sistema de análise de movimento } \\
\text { e plataforma de força }\end{array}$ \\
\hline$\underline{\text { Santos (1998) }}$ & 2 & $18 s, 22 s$ e $26 s$ & $31 \pm 2,82$ & $\begin{array}{l}\text { Cinética e } \\
\text { cinemática } \\
\text { marcha }\end{array}$ & $\begin{array}{c}\text { Sistema de análise de movimento } \\
\text { e plataforma de força }\end{array}$ \\
\hline Foti et al. (2000) & 15 & $3^{\circ} \operatorname{tr}$ e 1 ano $p p$ & 32 & $\begin{array}{c}\text { Cinética e } \\
\text { cinemática marcha }\end{array}$ & $\begin{array}{c}\text { Sistema de análise de movimento } \\
\text { e plataforma de força }\end{array}$ \\
\hline$\frac{\text { Gilleard, Crosbie e Smith }}{\underline{(2002)}}$ & $\begin{array}{l}9 \mathrm{G} \\
12 \mathrm{NG}\end{array}$ & $\begin{array}{c}18 \mathrm{~s} ; 24 \mathrm{~s} ; 32 \mathrm{~s} ; 38 \mathrm{~s} ; 8 \mathrm{~s} \\
\mathrm{pp}\end{array}$ & 28 a 40 & Controle postural & Sistema de análise de movimento \\
\hline$\underline{\text { Wu et al. (2002) }}$ & $\begin{array}{l}9 \mathrm{DP} \\
9 \mathrm{SD}\end{array}$ & NR & $\begin{array}{l}33,1 \\
33,4\end{array}$ & Cinemática marcha & $\begin{array}{c}\text { Sistema de análise de movimento } \\
\text { e acelerômetro }\end{array}$ \\
\hline Dunning et al. (2003) & 2847 & NR & NR & Quedas & Questionário \\
\hline Wu et al. (2004) & $\begin{array}{c}12 \mathrm{G} \\
13 \mathrm{NG}\end{array}$ & $20-34 s$ & 33,1 & Cinemática marcha & Sistema de análise de movimento \\
\hline Lymbery e Gilleard (2005) & 13 & $38 \mathrm{~s} ; 8 \mathrm{~s} p \mathrm{p}$ & 20 a 40 & Equilíbrio & Plataforma de força \\
\hline Butler et al. (2006a) & $\begin{array}{c}12 \mathrm{G} \\
12 \mathrm{NG}\end{array}$ & $\begin{array}{l}11-14 s ; 19-22 s \\
36-39 s ; 6-8 s p p\end{array}$ & $31,1 \pm 6$ & $\begin{array}{l}\text { Marcha e Equilíbrio } \\
\text { e quedas }\end{array}$ & $\begin{array}{c}\text { Sistema de análise de } \\
\text { movimento, plataforma de força e } \\
\text { questionário }\end{array}$ \\
\hline Butler et al. (2006b) & $\begin{array}{c}12 \mathrm{G} \\
12 \mathrm{NG}\end{array}$ & $\begin{array}{c}11-14 \mathrm{~s} ; 19-22 \mathrm{~s} ; 36- \\
39 \mathrm{~s} ; 6-8 \mathrm{spp}\end{array}$ & $\begin{array}{l}32,8 \pm 5 \\
31,1 \pm 6\end{array}$ & Equilíbrio e quedas & $\begin{array}{l}\text { Plataforma de força e } \\
\text { questionário }\end{array}$ \\
\hline$\frac{\text { Jang, Hsiao e Hsiao- }}{\underline{\text { Wecksler (2008) }}}$ & $\begin{array}{c}15 \mathrm{G} \\
15 \mathrm{NG} \\
\end{array}$ & $6 s ; 12 s ; 24 s p p$ & $31 \pm 4$ & Equilíbrio e quedas & $\begin{array}{l}\text { Plataforma de força } \\
\text { Questionário }\end{array}$ \\
\hline$\underline{\text { Carpes et al. (2008) }}$ & 7 & $\begin{array}{l}22-28 s ; 34-40 s ; \\
4 s p p\end{array}$ & 23 a 35 & Cinemática marcha & Sistema de análise de movimento \\
\hline Wu et al. (2008) & $\begin{array}{c}12 \\
12 \mathrm{DL} \\
\end{array}$ & $20-34 s$ & $\begin{array}{l}33,1 \\
33,5\end{array}$ & Cinemática marcha & Sistema de análise de movimento \\
\hline$\underline{\text { Mann et al. (2009) }}$ & $\begin{array}{c}13 \mathrm{G} \\
10 \mathrm{NG}\end{array}$ & $\begin{array}{c}13-16 s ; 22-25 s \\
28-29 s\end{array}$ & $\begin{array}{l}26,74 \pm 6,34 \\
19,7 \pm 1,43 \\
\end{array}$ & Equilíbrio e quedas & $\begin{array}{c}\text { Plataforma de força e } \\
\text { questionário }\end{array}$ \\
\hline Nagai et al. (2009) & $\begin{array}{l}35 \mathrm{G} \\
8 \mathrm{NG}\end{array}$ & $3^{\circ} \operatorname{tr}$ & $\begin{array}{l}30,6 \pm 0,6 \\
35,4 \pm 3,1 \\
\end{array}$ & Equilíbrio & Plataforma de força \\
\hline Oliveira et al. (2009) & 20 & $1^{\circ} ; 2^{\circ} ; 3^{\circ} \operatorname{tr}$ & $28,7 \pm 6,2$ & Equilíbrio & Plataforma de força \\
\hline
\end{tabular}

Onde: DL - dor lombar; DP - dor pélvica; SD - sem dor; s - semanas; pp - pós parto; NR - não referiu; G gestantes; NG - não gestantes; $\mathrm{tr}$ - trimestre.

\section{Discussão}

O presente estudo objetivou verificar o comportamento das variáveis cinéticas e cinemáticas da marcha humana e do controle da postura corporal durante a gestação, bem como verificar a ocorrência de quedas. Estudos recentes foram desenvolvidos para verificar características biomecânicas durante a gestação (CARPES et al., 2008; MANN et al., 2008b; MANN et al., 2009). Porém alguns pontos ainda continuam sem um consenso e precisam ser melhor investigados.

\section{Alterações posturais e marcha humana durante a gestação}

A marcha constitui-se em um movimento presente no dia-a-dia do ser humano, uma atividade complexa com padrões bem definidos. Durante a gestação, além de alterações posturais em decorrência da diminuição do equilíbrio corporal, estudos também têm investigado o alinhamento corporal, mudanças nos ângulos de flexão e extensão do quadril, joelho, coluna cervical e na marcha, dentre outras (GILLEARD, CROSBIE e SMITH, 2002; LYMBERY e GILLEARD, 2005; FOTI et al., 2000). 
As mudanças na estática e na dinâmica do esqueleto resultam, muitas vezes, em desconforto ou dor, causando limitações durante a realização das atividades da vida diária e profissional (MANN et al., 2008a). As dificuldades podem ser relacionadas às mudanças na mobilidade do tronco e no controle do movimento, devido ao aumento de massa e dimensões corporais. A justaposição de segmentos do corpo e dificuldades para controlar o aumento do momento angular, podem reduzir a gama funcional de movimentos dos segmentos do tronco (OSTGAARD et al., 1993).

Foti, Bagky e David (1998) estudaram a cinética e cinemática da marcha na segunda metade do terceiro trimestre de gestação e um ano após o parto. Foram encontrados aumentos significativos na anteversão pélvica, na flexão de quadril, adução de quadril, no tempo de duplo apoio; e uma significativa diminuição no tempo de apoio simples durante a gestação. Não foram relatadas mudanças significativas na base de suporte, comparadas aos momentos anteriores à gravidez. Os autores atribuem as mudanças suaves constatadas na pesquisa (dinâmica da base de suporte, limite de movimento da obliquidade pélvica e ângulo de progressão do pé) ao aumento da massa e largura do corpo, e à mudança na distribuição da massa sobre 0 tronco. Evidenciaram ainda que, durante a gravidez, pode haver uma demanda aumentada nos músculos abdutores e extensores do quadril, e músculo flexor plantar do tornozelo durante o caminhar.

A possível influência do aumento da massa corpórea e da distribuição desta no tronco sobre a cinética e cinemática da marcha também ficou evidenciada no estudo realizado por Foti et al. (2000) avaliando gestantes no terceiro trimestre e reavaliando-as um ano após o parto. Houve uma diminuição da flexão plantar durante a marcha (menor força de propulsão), aumento da abdução de quadril, aumento da inclinação pélvica (4ํㅜㄴ aproximadamente), mantendo a velocidade, 0 comprimento de passo, a cadência e os ângulos articulares relativamente inalterados, com aumento da demanda dos músculos abdutor e extensor do quadril e do flexor plantar do tornozelo.

Segundo Foti et al. (2000) e Nicholls e Grieve, (1992) muitos dos problemas músculoesqueléticos associados à gestação podem ser, em parte, associados a lesões músculoesqueléticas provocadas por sobrecargas ocorridas como conseqüência de desvios secundários da marcha que compensam as mudanças na massa e na distribuição da mesma pelo corpo. A ação hormonal, principalmente do hormônio relaxina é um fator a ser considerado, ele provoca o aumento do relaxamento articular e ligamentar, que torna as articulações mais flexíveis e, por exemplo, torna a região do quadril mais instável (BIRCH et al., 2003).

O principal fator biomecânico a ser considerado advém do constante crescimento do útero, sua posição anteriorizada dentro da cavidade abdominal, além do aumento no peso e no tamanho das mamas, que contribuem para o deslocamento do centro de gravidade da mulher para cima e para frente, podendo acentuar a lordose lombar e promover uma anteversão pélvica (CONTI, CALDERON e RUDGE, 2003). O útero ganha aproximadamente seis quilos até o final da gestação e seu desenvolvimento resulta em uma protusão abdominal, deslocamento superior do diafragma, mudanças compensatórias na mecânica da coluna vertebral e rotação pélvica (GAZANEO e OLIVEIRA, 1998). Buscando compensar essa hiperlordose lombar e manter a linha de visão, a gestante aumenta a flexão anterior da coluna cervical, anteriorizando a cabeça, hiperestendendo os joelhos, alargando a base de suporte e transferindo o peso para a região dos calcâneos (WINTER, 1995; WANG e APGAR, 1998).

Segundo Santos (1998) as maiores alterações de massa e de forma ocorrem no tronco inferior, que por sua vez está diretamente ligado às articulações do membro inferior. O ganho de massa nos segmentos, principalmente no tronco, leva a alteração do valor inercial desse segmento, bem como dos demais. Estas mudanças podem gerar modificações nas acelerações do centro de massa do corpo, sendo que estas estão diretamente ligadas às forças de reação do solo, ainda que somente parte da carga seja diretamente devido ao peso corporal, indiretamente, 0 fator peso do corpo pode ter influenciado a mecânica das articulações do membro inferior modificando a função da marcha e do equilíbrio dessas gestantes. A partir deste ganho de peso, as articulações do quadril, joelho e tornozelo são afetadas através dos fatores gravitacionais e de aceleração. Com isso, os movimentos devem ser adaptados e os momentos inter-segmentares modificados para se adequar a forma e inércia do tronco inferior, gerando ajustes tanto na postura como na marcha (SANTOS, 1998).

Em relação a distribuição de massa corpórea durante a gestação Jensen, Doucet e Treitz (1996) afirmam que o momento dos movimentos 
do quadril, principalmente flexão e extensão, aumentam conforme 0 avanço do período gestacional, sugerindo que o aumento de massa tem um efeito substancial nestas variáveis, que estão relacionadas com a dinâmica da marcha. Para chegar a estas conclusões os autores avaliaram as mudanças na massa estimada e os principais momentos inerciais dos segmentos corporais de gestantes durante o segundo e terceiro trimestres de gestação. Encontraram um aumento na massa do tronco inferior de $0,29 \mathrm{~kg}$ por semana e de $0,0069 \mathrm{~kg} \cdot \mathrm{m}^{2}$ por semana. Porém os autores sugerem a realização de outros trabalhos para predizer de forma mais efetiva os momentos inerciais através de um modelo de regressão (JENSEN, DOUCET e TREITZ, 1996).

Paul, Salk e Frings-Dresen (1996) calcularam o momento resultante no quadril através da combinação deste fator com o peso segmentar e com o centro de massa do tronco de gestantes. Entre a $10^{\text {a }}$ e a $40^{\text {a }}$ semanas de gestação o momento aumentou $52 \mathrm{~N} . \mathrm{m}$, isto é, a carga na $40^{\text {a }}$ semana de gestação foi 2,8 vezes maior do que na $10^{\underline{a}}$ semana. Em média, a metade deste aumento foi causada pelas mudanças posturais. Mudanças na massa segmentar, no centro de massa do tronco, e a interação entre os três fatores foram responsáveis por 10, 20 e 15\% do aumento da carga, respectivamente. $\mathrm{O}$ aumento no momento de reação do quadril pode ser minimizado prevenindo as mudanças posturais adversas, e otimizando a postura para reduzir a sua contribuição para as mudanças na distribuição do peso corporal.

Avaliando as variáveis espaço-temporais e cinemáticas durante a marcha, Santos (1998) encontrou que as variáveis cinemáticas demonstram uma tendência na redução do comprimento do passo e comprimento da passada, na cadência, no tempo de apoio simples, e tendência a aumento no tempo de duplo apoio e na base de suporte quando se observa os dados de uma semana para a outra. Segundo o autor, o aumento do tempo de duplo apoio e diminuição no tempo de apoio simples são estratégias para prevenir eventuais problemas de equilíbrio e de cinestesia advindos do ganho de peso. Já as variáveis cinéticas (primeiro e segundo picos de força, força média, impulso) apresentaram uma tendência na elevação de seus valores no transcorrer da gestação, sendo que as maiores diferenças foram encontradas entre a $18^{\mathrm{a}}$ e a $22^{\mathrm{a}}$ e $18^{\mathrm{a}}$ e a $26^{\mathrm{a}}$ semanas, explicadas também pelo ganho de peso, já que as reações do solo ou do piso comum são uma resposta às ações musculares e ao peso do corpo, transmitidos pelos pés. Há uma maior necessidade de flexão do joelho com a evolução da gestação a fim de se obter uma maior suavização das alterações bruscas nas intersecções dos arcos das trajetórias dos centros de massa do membro inferior e principalmente na suavização da trajetória do quadril muito sobrecarregado devido as modificações posturais impostas pela gestação (SANTOS, 1998).

Segundo Santos (1998) as mudanças que ocorrem na forma do corpo da mulher grávida trazem maior dificuldade na realização dos movimentos, além de propiciar um estado de fadiga mais rápido, bem como, maior esforço muscular devido ao ganho de massa. Todas estas alterações são acompanhadas por uma diminuição significativa na freqüência de passada e ajustes posturais. Lymbery e Gilleard (2005) evidenciaram aumento da largura do passo e da força de reação do solo (FRS) no sentido médio lateral. Segundo os autores essas diferenças sugerem que as gestantes adaptam seu padrão de andar para maximizar a estabilidade e controlar movimentos médio-laterais.

No recente estudo de Carpes et al. (2008) foi investigada a marcha de gestantes. Os resultados demonstram que duplo apoio, apoio simples e tempo de apoio diferiram estatisticamente entre P1 (22 a 28 semanas) e P2 (34 a 40 semanas); P1 e P3 (após o parto), mas não entre P2 e P3. As variáveis comprimento do passo e comprimento da passada apresentaram diferenças significativas apenas entre P1 e P2. Já em relação as variáveis angulares, não foram encontradas diferenças no ângulo de flexãoextensão do quadril, a média de flexão do quadril foi menor em P1 que em P2 e menor em P2 que em $\mathrm{P} 3$, indicando um progressivo aumento da flexão de quadril com o avançar da gestação.

O mesmo ocorreu durante 10, 20,30,40, 50, 90 e $100 \%$ do ciclo da marcha, com a flexão de quadril sendo maior em P3. Em $60 \%$ do ciclo de passada a flexão de quadril foi maior em P2 comparado aos demais períodos, enquanto que em 70 e $80 \%$ foi maior em P1. Aumento do ângulo de flexão e extensão do joelho, a $40 \%$ a diferença foi entre P1 e P2 e entre P2 e P3. A $50 \%$ a diferença foi apenas entre P2 e P3, sem diferenças entre os outros períodos. Aumento da mobilidade articular, sendo que nas variáveis espaço-temporais permaneceram até 4 meses após o parto (P3), enquanto a cinemática angular da marcha no $3^{\circ}$ período (P3) foi similar a marcha normal (CARPES et al., 2008).

Butler et al. (2006a) verificaram que as medidas cinéticas e cinemáticas do andar de 
gestantes permanecem relativamente estáveis durante a gravidez, comparadas a valores de sujeitos normais. Aumentos significantes na fase final da extensão do quadril e flexão de joelho foram encontrados e associados com um aumento do momento do músculo extensor do joelho; aumento do pico da dorsiflexão de tornozelo no final da marcha, das grávidas no terceiro trimestre, comparado a valores do grupo controle. Aumentos na extensão, adução e rotação interna do quadril, na fase inicial da gravidez, também foram percebidos e permaneceram aumentados após o parto.

A grande incidência de dor lombar e pélvica durante a gestação já foi destacada em estudos anteriores (MANN et al., 2008a; WU et al., 2008). A ocorrência de dor lombar e pélvica é considerada uma das principais síndromes advindas do desenvolvimento da gestação, atingindo mais de um terço das gestantes (WU et al., 2004), principalmente a partir do $3^{\circ}$ trimestre do período gestacional (MANN et al., 2008a). A prática de terapias e exercícios físicos é apontada como fator de redução e prevenção das dores (MANN et al., 2008a).

Devido a isso, alguns estudos têm investigado a coordenação na rotação pélvica e do tronco durante a gestação (WU et al., 2002; 2004; 2008). Wu et al. (2002) encontraram que gestantes com dor pélvica tem prejuízos na realização da marcha devido a problemas de coordenação na rotação de pélvis e tronco. As rotações pélvicas e torácicas das gestantes com dor pélvica foram significativamente mais sincronizadas (rotações na mesma direção) e a relação entre estas rotações foi significativamente mais forte. Encontraram também que as gestantes com dor lombar apresentaram velocidade média da marcha menor que as gestantes que não possuíam dor, mas com maior variação entre as gestantes patológicas do que nas saudáveis.

Já Wu et al. (2008) encontraram que a velocidade da marcha foi menor nas gestantes com dor em relação as sem dor e aumento da rotação pélvica com dor. As amplitudes de rotação foram maiores com presença de dor, e o pico de rotação do tórax ocorreu primeiro. O grupo com dor apresentou marcha com menor velocidade que o grupo controle, e negativamente relacionada com 0 medo do movimento. Enquanto que as amplitudes rotacionais das gestantes com dor foram maiores, com grandes diferenças inter-individuais, as rotações da coluna não diferiram entre os grupos. Nas gestantes acometidas, o pico máximo de rotação do tórax ocorreu mais cedo durante o ciclo do passo a velocidades mais altas, e esta fase relativa foi menor. Rotações espinhais permaneceram não afetadas, enquanto em velocidades mais altas o pico de rotação do tórax aconteceu mais cedo durante o ciclo de passo. A última mudança pode servir para evitar rotações excessivas da coluna causadas por rotações segmentárias maiores.

Investigando a influência da gestação sobre a rotação da pélvis e tórax $\mathrm{Wu}$ et al. (2004) encontraram que a rotação pélvica foi menor nas gestantes em comparação a não gestantes, e a amplitude de rotação da pélvis, tórax e tronco foi menor nas gestantes. Segundo os autores, as gestantes tendem a evitar a rotação pélvica e torácica em altas velocidades, possivelmente porque o aumento nos momentos de inércia da pélvis e tórax torna o controle desta fase relativamente mais crítico.

Durante tarefas diárias o alinhamento postural também pode sofrer alterações, dessa forma, Gilleard, Crosbie e Smith (2002) investigaram o alinhamento postural do tronco no plano sagital durante posturas de sentar e levantar. Não foram encontrados efeitos significantes da gestação na postura superior do corpo, embora houvesse uma tendência em alguns sujeitos para uma diminuição da curvatura lombar na posição sentada com a progressão da gestação. Em situação estática após o parto, a pelve teve uma redução na sua orientação anterior, e a coluna lombar estava menos estendida, indicando uma retificação da curvatura espinhal quando comparado com o grupo controle. O alinhamento no plano sagital durante a postura em pé dos segmentos tórax e cabeça foram levemente posteriorizados, e ocorreu aumento da extensão da região toraco-lombar e cervico-torácica em relação ao grupo controle de não gestantes. Não foram encontradas diferenças significativas com o avançar da gestação no alinhamento da pélvis, tórax e cabeça e nas regiões toraco-lombar e toraco-cevical, como também não foram encontradas diferenças na avaliação pós-parto.

Gestantes com problemas musculoesqueléticos deveriam enfatizar $o$ valor do exercício e do condicionando físico durante a gravidez, pois ambos auxiliam na prevenção e na reabilitação (FOTI et al., 2000).

\section{Equilíbrio corporal e quedas durante o processo gestacional}

Além das alterações específicas na postura (alinhamento dos segmentos corporais, alterações cinemáticas) já mencionadas no tópico acima, o estudo da postura através da análise de oscilação do centro de força (equilíbrio) é um 
tema de crescente interesse entre os estudiosos (FRANKLIN e CONNER-KERR, 1998; BUTLER et al., 2006a; HUE et al., 2007; JANG, HSIAO e HSIAO-WECKSLER, 2008; OLIVEIRA et al., 2009; MANN et al., 2009) principalmente na ultima década, fato que se confirma pela concentração dos estudos a partir de 2000.

Isso se deve a importância do equilíbrio e da necessidade de seu correto e perfeito funcionamento durante uma infinidade de atividades realizadas pelo ser humano durante atividades da vida diária, durante 0 andar, durante o alcance de um objeto com as mãos, quando nos comunicamos ou alimentamos, ou mesmo quando decidimos ficar parados em pé, sentados ou deitados.

De acordo com Horak e Macpherson (1996) o equilíbrio postural está relacionado ao controle da relação entre forças externas (força gravitacional), que agem sobre o corpo, e forças internas (torques articulares), que são produzidas pelo corpo. Este controle é necessário, pois as forças que atuam no corpo e/ou são produzidas pelo corpo agem acelerando-o e, conseqüentemente, fazendo com que este altere seu alinhamento e se afaste da posição desejada ou da posição considerada de maior estabilidade. Desta forma, o equilíbrio corporal é alcançado quando todas as forças que agem neste corpo, tanto externas como internas, estão controladas, o que permite que o corpo permaneça em uma posição desejada (equilíbrio estático) ou que se mova de uma maneira controlada (equilíbrio dinâmico).

A manutenção do equilíbrio do corpo segundo Horak e Macpherson, (1996) atribuída ao sistema de controle postural, se refere às funções dos sistemas nervoso, sensorial e motor para 0 desempenho dessa tarefa.

O sistema motor é responsável pela ativação correta e adequada de músculos para realização dos movimentos através de ações coordenadas dos vários grupos musculares, responsáveis pela manutenção da relação estabelecida entre os segmentos corporais e da relação do conjunto destes segmentos, ou do corpo como um todo, com o ambiente (FREITAS Jr. e BARELA, 2006).

O sistema sensorial através dos estímulos captados pelos sistemas vestibular, somatossensorial e visual e enviados ao SNC e integrados no sistema de controle postural fornece informações sobre estímulos sensoriais captados continuamente durante a manutenção da postura ereta sobre a posição de segmentos corporais em relação a outros segmentos e ao ambiente (HORAK e MACPHERSON, 1996).
Ao sistema vestibular cabe a sensibilidade de detectar as acelerações lineares e angulares, enquanto o sistema somatossensorial é composto por vários receptores (órgãos tendinosos de Golgi, receptores articulares e cutâneos e fusos musculares) que percebem a posição e a velocidade de todos os segmentos corporais, seu contato com objetos externos, inclusive o chão, e a orientação da gravidade (WINTER, 1995). Já a informação visual é baseada nas informações externas do ambiente e da direção e velocidade dos movimentos corporais em relação ao ambiente, além de diferenciar o que é automovimento, ou movimento do próprio corpo, do que é movimento de um objeto no ambiente (HORAK e MACPHERSON, 1996).

Já ao sistema nervoso central cabe integrar as informações provenientes do sistema sensorial para então enviar impulsos nervosos aos músculos que geram respostas neuromusculares (HORAK e MACPHERSON, 1996). Devido a isso, estudiosos de diversas áreas buscam verificar se com o desenvolvimento da gestação ocorre alguma alteração nesse integrado e complexo sistema, que seja capaz de provocar maiores oscilações do corpo da gestante, fazendo com que ela apresente seu equilíbrio próximo aos limites de estabilidade.

Dessa forma é preciso considerar alguns pontos importantes ao analisar o equilíbrio como, altura do centro de gravidade, tamanho da base de apoio ou suporte, utilização e importância dos sistemas sensoriais (visual, somatossenssorial e vestibular) dentre outros. Uma mudança ou mau funcionamento em algum desses aspectos pode provocar alterações significativas.

Franklin e Conner-Kerr (1998) investigaram o equilíbrio corporal no primeiro e no terceiro trimestre da gestação. Os autores encontraram significativas alterações no controle corporal no terceiro trimestre em comparação ao primeiro, além de uma diminuição da cifose cervical e inclinação pélvica.

Butler et al. (2006a) encontraram que o equilíbrio corporal foi diminuindo no segundo e terceiro trimestre de gestação, comparado a valores de não gestantes e, permaneceu diminuindo após o parto (6 a 8 semanas). A diferença nos valores de equilíbrio ao se analisar a influência da visão (olhos abertos e olhos fechados) foram maiores no terceiro trimestre comparados aos valores de não gestantes e continuaram aumentando com o progresso da gravidez, sendo a condição com ausência da visão a com os maiores valores de oscilação. $O$ mesmo foi reportado por Butler et al. (2006b) que 
encontraram aumento nos desequilíbrios corporais com o avançar da gestação, tanto com os olhos abertos quanto com os olhos fechados, permanecendo diminuídos após o parto.

Segundo Butler et al. (2006b) o ganho de peso mais gradual característico do primeiro trimestre da gestação, explica as não diferenças no equilíbrio corporal no primeiro trimestre gestacional em comparação a não gestantes. A partir do segundo e terceiro trimestres ocorre um ganho de peso maior o que pode explicar a diminuição em estabilidade postural durante essas fases da gestação. Além disso, o aumento da altura do centro de gravidade e a frouxidão ligamentar principalmente da região do quadril combinados provocam as alterações no equilíbrio durante a gestação. Com o ganho de peso característico da gestação, o centro de massa do corpo move-se para frente, e assim, afeta a estabilidade postural em resposta às perturbações que ocorrem pela relação entre a posição do centro de massa e a resposta do torque do tornozelo a este movimento. Com o ganho de massa do corpo, ocorre uma diminuição na habilidade dos mecano-receptores em receber as sensações cutâneas, fazendo com que as pressões e forças sejam maiores (Força de Reação do Solo), reduzindo a captação da informação sensorial e causando maiores instabilidades corporais (HUE et al., 2007).

Duarte (2000) afirma que com o ganho de peso aumenta-se 0 torque necessário para manter o equilíbrio, causando um aumento na atividade muscular envolvida para atuar motoramente na tarefa de trazer o centro de massa de volta para dentro da base de apoio, o que aumenta os valores das variáveis de oscilação, já que o corpo em posição ereta é freqüentemente comparado com um sistema de pêndulo, movendo-se pelo eixo dos tornozelos (DUARTE, 2000). Durante a gestação essa distribuição anormal de massa, fica evidenciada principalmente na região do abdome, causando, por exemplo, um desequilíbrio para frente, devendo haver uma resposta necessária de torque do tornozelo, para recuperar o equilíbrio, de magnitude muito alta.

Da mesma forma que em estudos anteriores, diferenças significativas no equilíbrio foram relatadas a partir da $20^{\text {a }}$ semana de gestação quando comparadas a não gestantes no estudo de Jang, Hsiao e Hsiao-Wecksler (2008). Durante a gestação ocorreu aumento da oscilação corporal na direção ântero-posterior diminuindo os valores de oscilação após o parto. Na direção médio-lateral as oscilações permaneceram estáveis durante a gravidez, mas aumentadas após o parto. Segundo os autores as não diferenças na direção médio-lateral reportadas em seu estudo são devido ao aumento da base de apoio durante a gestação para manutenção da estabilidade. $\mathrm{O}$ aumento da base de apoio variou entre $17,9 \mathrm{~cm}$ e $20,6 \mathrm{~cm}$ entre a $16^{\text {a }}$ e a $36^{\text {a }}$ semanas de gestação, chegando a $21,9 \mathrm{~cm}$ na $40^{\mathrm{a}}$ semana de gestação. Logo após o parto houve uma diminuição para $17,6 \mathrm{~cm}$ e esta foi para $17,7 \mathrm{~cm}$ na $24^{\mathrm{a}}$ semana após o parto.

Um aspecto importante mencionado no estudo de Jang, Hsiao e Hsiao-Wecksler (2008) é a modificação da base de apoio e da manipulação da visão durante a gestação e após o parto, atuando como estratégias do sistema de controle postural para manter a estabilidade corporal e evitar as quedas (BIRD et al., 1999; FOTI et al., 2000; LYMBERY e GILLEARD, 2005; JANG; HSIAO; HSIAO-WECKSLER, 2008). Santos (1998) evidenciou uma tendência de aumento da base de suporte, provavelmente buscando um melhor equilíbrio do corpo bem como um menor esforço muscular a fim de gerar um menor gasto energético.

A influência da modificação do posicionamento dos pés também é um fator considerado nos estudos. Oliveira et al. (2009) avaliaram 20 gestantes em 4 condições sendo, olhos abertos e pés separados confortavelmente; olhos fechados e pés separados confortavelmente; olhos abertos e pés juntos; olhos fechados e pés juntos nos três trimestres de gestação. O tamanho da base de suporte não diferiu estatisticamente com 0 avançar da gestação. Mudanças significativas no equilíbrio corporal com o avançar da gestação (segundo e terceiro trimestres) foram observadas principalmente com os pés afastados confortavelmente e olhos fechados. A área de oscilação aumentou significativamente com o avançar da gestação nos protocolos com os pés separados e olhos fechados. A análise espectral revelou um significante aumento de oscilações do centro de força (COP) ao longo da direção ântero-posterior quando os sujeitos estavam com os olhos fechados e pés juntos.

Segundo Oliveira et al. (2009) as gestantes tendem a compensar a situação de falta de visão aumentando a freqüência de oscilação do COP nas direções ântero-posterior e médio-lateral ao longo da gestação. Os resultados indicam uma maior confiança na contribuição visual para controle de equilíbrio durante fases mais avançadas de gravidez. Resultados semelhantes foram reportados por Nagai et al. (2009) 
investigando 35 gestantes no último trimestre da gestação. Os resultados demonstram diferença significativa na área do COP nas gestantes em comparação às não gestantes e na variável comprimento da trajetória no sentido médiolateral e ântero-posterior com os olhos fechados, denotando segundo os autores uma dependência nas informações advindas dos sistemas somatossensorial e visual para manutenção do equilíbrio com o avançar da gestação.

Contrariando os estudos acima que referem dependência da informação proveniente do sistema visual, Mann et al. (2009) encontraram que gestantes tem diminuída sua habilidade de manter o equilíbrio com avançar da gestação, porém sem diferenças significativas nas instabilidades corporais com a ausência da visão. As não diferenças em relação ao grupo controle foram observadas apenas no grupo inicial (13 a 16 semanas), em ambas as condições sensoriais, o que indica que o início do período gestacional não é o mais acometido pelos sintomas de desequilíbrios. Segundo os autores, o aumento de oscilação corporal pode estar relacionado a uma adaptação neuromuscular ou a uma estratégia compensatória para manter a postura ereta em uma situação onde a projeção vertical do centro de gravidade se encontre próximo aos limites da base de apoio, como ocorre durante a gestação.

A principal conseqüência apontada na literatura advinda de problemas de equilíbrio são as quedas (DUNNING et al., 2003; BUTLER et al., 2006a). As quedas surgem como uma forte problemática resultante dessas alterações no equilíbrio e, segundo alguns autores (DUNNING et al., 2003; BUTLER et al., 2006a) são eventos comuns durante a gestação.

Butler et al. (2006a; 2006b) encontraram que $1 / 4$ das gestantes tiveram quedas durante a gravidez. Dunning et al. (2003) encontraram a mesma proporção de quedas que o estudo anterior a qual é comparável a pessoas idosas com idade superior a 65 anos. Foram avaliadas 2.847 gestantes, destas 26,6\% (757) caíram durante a gestação, sendo que 6,3\% (179) caíram durante o trabalho, em situações com pisos molhados ou escorregadios, e carregando objetos. No estudo de Jang. Hsiao e HsiaoWecksler (2008) 13\% das gestantes avaliadas relataram quedas durante o período gestacional. Contrariando os demais estudos Mann et al. (2009) não relataram quedas durante a gestação.

As principais causas ou limitações para essas lacunas persistirem são principalmente:
- A falta de um acompanhamento em um grupo de gestantes que inicie logo nas primeiras semanas de gestação e que possa dar um diagnóstico mais preciso sobre as mudanças musculoesqueléticas e decorrentes alterações no padrão da marcha e da postura, com um menor intervalo entre as avaliações;

- A falta de uma progressão nas avaliações dificulta $o$ entendimento das mudanças ocorridas em cada fase da gestação sobre as características da postura e da marcha.

\section{Conclusão}

A presente revisão de literatura trouxe informações importantes a respeito das alterações músculo esqueléticas advindas do processo gestacional, como:

- Mudanças significativas no equilíbrio corporal começam a ser observadas a partir do segundo trimestre gestacional e geralmente permanecem assim algumas semanas após o parto. A supressão da visão aumenta os desequilíbrios e o aumento ou modificação da base de apoio são observados na gestação e deve-se tomar cuidado com estes fatores;

- As quedas ocorrem na ordem de $1 / 4$ das gestações;

- $\mathrm{Na}$ realização da marcha evidencia-se mudanças significativas na cinética e cinemática no membro inferior advindas das mudanças na distribuição de massa como aumentos na flexão, extensão, adução e abdução do quadril, flexão e extensão do joelho e dorsiflexão de tornozelo além de aumento no tempo de apoio duplo, diminuição no tempo de apoio simples, aumento nas variáveis cinéticas $\left(1^{\circ}\right.$ e $2^{\circ}$ picos de força e impulso) com o avançar da gestação;

- Em relação ao alinhamento corporal percebe-se aumento na lordose lombar e da anteversão pélvica.

Apesar de vários aspectos já serem fortemente relacionados como consequências do processo gestacional sugere-se a realização de estudos futuros que avaliem um grupo significativo de gestantes, em que se façam avaliações mensais, com controle de variáveis como circunferência da cintura (diâmetro da barriga), ganho de peso, localização do centro de massa, tamanho da base de apoio, tamanho do pé, presença de dor lombar ou pélvica, lordose, cifose, escoliose prévias a gestação, prática de exercício físico, idade, número de gestações etc., que são fatores que podem intervir para uma boa postura e marcha. Dessa forma será possível minimizar problemas e sugerir métodos de intervenção precoces e eficazes, pois haverá um controle intenso de possíveis fatores intervenientes. 


\section{Referências}

BIRCH, K.; FOWLER, N. E.; RODACKI, A. L.; RODACKI, C. L. Stature loss and recovery in pregnant women with and without low back pain. American Academy physical medicine and rehabilitation, Chicago, v. 84, n. 4, p. 507-512, 2003. http://dx.doi.org/10.1053/apmr.2003.50119.

BIRD, A. R.; MENZ, H. B.; HYDE, C. C. The effect of pregnancy on footprint parameters. $A$ prospective investigation. Journal of the American Podiatric Medical Association, Maryland, v. 89, n. 8, p. 405-409, 1999.

Disponível em:

http://www.japmaonline.org/cgi/reprint/89/8/405.

Acesso em: 03 jun. 2008.

BUTLER, E. E.; COLÓN, I.; DRUZIN, M.; ROSE, J. Postural equilibrium during pregnancy: Decreased stability with an increased reliance on visual cues. American journal of obstetrics and gynecology, Nova York, v. 195, n. 4, p. 11041108, 2006a.

http://dx.doi.org/10.1016/..ajog.2006.06.015.

BUTLER, E. E.; COLÓN, I.; DRUZIN, M.; ROSE, $\underline{\mathrm{J}}$. An investigation of gait and postural balance during pregnancy. Gait and Posture, Amsterdam, v. 24, (suppl. 2), p. 128-129, 2006 b.

http://dx.doi.org/10.1016/i.gaitpost.2006.11.089.

CARPES, F. P.; GRIEBELER, D.; KLEINPAUL, J. F.; MANN, L.; MOTA, C. B. Women able-bodied gait kinematics during and post pregnancy period. Revista Brasileira de Biomecânica, São Paulo, v. 9, n. 16, p. 33-40, 2008. Disponível em: http://143.107.170.186//ojs/index.php/rbb. Acesso em: 12 nov. 2008.

CONNOLLY, A. M.; KATZ, V. L.; BASH, K. L.; MCMAHON, M. J.; HANSEN, W. F. Trauma and pregnancy. American journal of perinatology, New York, v. 14, p. 331-336, 1997.

http://dx.doi.org/10.1055/s-2007-994155.

CONTI, M. H. S; CALDERON, I. M. P.; RUDGE, M. V. C. Desconfortos músculo-esqueléticos da gestação - uma visão obstétrica e fisioterápica. Femina, São Paulo, v. 31, n. 6, p. 531-535, 2003. Disponível em:

http://www.febrasgo.org.br/?op=paginas\&tipo=pa gina\&secao $=8 \&$ pagina $=50$. Acesso em: 12 nov . 2008.

DUNNING, K.; LEMASTERS, G.; LEVIN, L.; BHATTACHARYA, A.; ALTERMAN, T.; LORDO, K. Falls in workers during pregnancy: risk factors, job hazards, and high risk occupations. American journal of industrial medicine, v. 44, n. 6, p. 664-672, 2003.

http://dx.doi.org/10.1002/ajim.10318.
DUARTE, M. Análise estabilográfica da postura ereta humana quasi-estática. 2000. 87f. Tese (Doutorado em Educação Física e Esporte) - Departamento de Biodinâmica do Movimento do Corpo Humano, Universidade de São Paulo, São Paulo, 2000. Disponível em: http://demotu.org/pubs/duarte00.pdf. Acesso em: 10 nov. 2008.

FOTI, T.; BAGKY, A.; DAVID, J. Biomechanical attentions in gait during pregnancy. Gait and Posture, Amsterdam, v. 7, p. 169-170, 1998. Disponível em:

http://www.elsevier.com/wps/find/journaldescriptio n.cws home/525442/description\#description. Acesso em: 10 jun. 2000.

\section{FOTI, T.; DAVIDS, J. R.; BAGLEY, A. A}

biomechanical analysis of gait during pregnancy. The Journal of bone and joint surgery, Illinois, v. 82 , n. 5, p. 625-632, 2000. Disponível em: http://www.ejbjs.org/cgi/reprint/82/5/625. Acesso em: 03 jun. 2008.

FRANKLIN, M. E.; CONNER-KERR, T. An analysis of posture and back pain in the first and third trimesters of pregnancy. The Journal of orthopaedic and sports physical therapy, v. 28, n. 3, p. 133-138, 1998. Disponível em:

http://www.ncbi.nlm.nih.gov/pubmed/9742469.

Acesso em: 10 jan. 2008.

FREITAS JÚNIOR, P.; BARELA, J. A. Alterações no funcionamento do sistema de controle postural de idosos. Uso da informação visual. Revista Portuguesa de Ciência do Desporto, Porto, v. 6, n. 1, p. 94-105, 2006. Disponível em: http://www.fade.up.pt/rpcd/entrada.html. Acesso em: 23 jun. 2007.

GAZANEO, M. M.; OLIVEIRA, L. F. Alterações posturais durante a gestação. Revista Brasileira de Atividade Física \& Saúde, Londrina, v. 3, n. 2, p. 13-21, 1998.

http://www.sbafs.org.br/revista/revistas.php. Acesso em: 12 ago. 2008.

GILLEARD, W. L.; CROSBIE, J.; SMITH, R. Static Trunk Posture in Sitting and Standing During Pregnancy and Early Postpartum. Archives Physical Medicine Rehabilitation, Chicago, v. 83, n. 2, p. 1739-1744, 2002.

http://dx.doi.org/10.1053/apmr.2002.36069.

HORAK, F. B.; MACPHERSON, J. M. Postural orientation and equilibrium. In: ROWELL, L. B.; SHERPHERD, J. T. (ed.) Handbook of physiology: a critical, comprehensive presentation of physiological knowledge and concepts. New York: Oxford American Physiological Society, 1996. p. 255-292. 
HUE, O.; SIMONEAU, M.; MARCOTTE, J.; BERRIGAN, F.; DORÉ, J.; MARCEAU, P.; MARCEAU, S.; TREMBLAY, A.; TEASDALE, N. Body weight is a strong predictor of postural stability. Gait and Posture, Amsterdam, v. 26, n. 1, p. 32-38, 2007.

http://dx.doi.org/10.1016/j.gaitpost.2006.07.005.

JANG, J.; HSIAO, K. T.; HSIAO-WECKSLER, E. $\underline{T}$. Balance (perceived and actual) and preferred stance width during pregnancy. Clinical

Biomechanics, Oxford, v. 23, n. 4, p. 468-476, 2008.

http://dx.doi.org/10.1016/j.clinbiomech.2007.11.01 1 .

JENSEN, R. K.; DOUCET, S.; TREITZ, T. Changes in segment mass and mass distribution during pregnancy. Journal of Biomechanics, Oxford, v. 29, n. 2, p. 251-256, 1996. http://dx.doi.org/10.1016/0021-9290(95)00042-9.

LYMBERY, J. K.; GILLEARD, W. The stance phase of walking during late pregnancy: temporospatial and ground reaction force variables. Journal of the American Podiatric Medical Association, Maryland, v. 95, n. 3, p. 247-253, 2005. Disponível em:

http://www.japmaonline.org/cgi/reprint/95/3/247. Acesso em: 3 fev. 2009.

MANN, L.; KLEINPAUL, J. F.; TEIXEIRA, C. S.; KONOPKA, C. K. Dor lombo-pélvica e exercício físico durante a gestação. Fisioterapia em Movimento, Curitiba, v. 21, n. 2, p. 99-105, 2008a. Disponível em:

http://www2.pucpr.br/reol/index.php/RFM?dd1=19 44\&dd99=view. Acesso em: 1 jan. 2008.

MANN, L.; KLEINPAUL, J. F.; TEIXEIRA, C. S.; MOTA, C. B. A marcha humana: investigação com diferentes faixas etárias e patologias. Motriz, Rio Claro, v. 14, n. 3, p. 346-353, 2008b.

Disponível em:

http://cecemca.rc.unesp.br/ojs/index.php/motriz/is sue/view/750. Acesso em: 3 fev. 2009.

MANN, L.; KLEINPAUL, J. F.; TEIXEIRA, C. S.; LOPES, L. F. D.; KONOPKA, C. K.; MOTA, C. B. Gestação: equilíbrio corporal, dor lombar $e$ quedas. Brazilian Journal of Biomechanics, São Paulo, v. 9, n.18, p. 14- 21, 2009. Disponível em: http://143.107.170.186/ojs/index.php/rbb. Acesso em: 1 jul. 2009.

MANTLE, J.; POLDEN, M. Fisioterapia em ginecologia e obstetrícia. São Paulo: Editora Santos, 2005.

MARNACH, M. L.; RAMIN, K. D.; RAMSEY, P. S.; SONG, S. W.; STENSLAND, J. J.; AN, K. N. Characterization of the relationship between joint laxity and maternal hormones in pregnancy.
European journal of obstetrics, gynecology, and reproductive biology, Ireland, v. 101, n. 2, p. 331-335, 2003. Disponível em:

http://www.labmeeting.com/paper/24840731/marn ach-2003-characterization-of-the-relationshipbetween-joint-laxity-and-maternal-hormones-inpregnancy. Acesso em: 10 dez. 2008.

NAGAI, M.; ISIDA, M.; SAITOH, J.; HIRATA, Y.; NATORI, H.; WADA, M. Characteristics of the control of standing posture during pregnancy. Neuroscience Letters, New Haven, v. 462, p. 130-134, 2009.

http://dx.doi.org/10.1016/j.neulet.2009.06.091.

NICHOLLS, J. A.; GRIEVE, D. W. Posture, performance and disconfort in pregnancy. Applied Ergonomics, Oxford, v. 23, n. 2, p. 128132, 1992. http://dx.doi.org/10.1016/00036870(92)90085-A.

OLIVEIRA, L. F.; VIEIRA, T. M. M.; MACEDO, A. R.; SIMPSON, D. M.; NADAL, J. Postural sway changes during pregnancy: A descriptive study using stabilometry. European Journal of Obstetrics \& Gynecology and Reproductive Biology, Ireland, p. 1-4, 2009.

http://dx.doi.org/10.1016/i.ejogrb.2009.06.027.

OSTGAARD, H. C.; ANDERSSON, G. B.; SCHULTZ, A. B.; MILLER, J. A. Influence of some biomechanical factors on low-back pain in pregnancy. Spine, Philadelphia, v. 18, p. 61-65, 1993. Disponível em:

http://journals.Iww.com/spinejournal/Abstract/1993 101000/Influence of Some Biomechanical Facto rs on.10.aspx. Acesso em: 18 de set. 2008.

PAUL, J. A.; SALK, H.; FRINGS-DRESEN M, H. $\underline{W}$. Effect of posture on hip joint moment during pregnancy, while performing a standing task. Clinical Biomechanics, Oxford, v. 11, n. 2, p. 111-115, 1996. http://dx.doi.org/10.1016/02680033(95)00049-6.

\section{SANTOS, G. M. Avaliação biomecânica do} andar durante a gestação. 1998. $79 \mathrm{f}$. Dissertação (Mestrado em Ciência do Movimento Humano) - Centro de Educação Física e Desportos, Universidade Federal de Santa Maria, Santa Maria, 1998.

WANG, T. W.; APGAR, B. S. Exercise during Pregnancy. American Family Physician, [SI], v. 57, n. 8, p. 1846-1852, abr. 1998.

WEISS, H. B.; SONGER, T. J.; FABIO, A. Fetal deaths related to maternal injury. The journal of the American Medical Association, v. 286, n. 15, p. 1863-1868, 2001. Disponível em: http://jama.amaassn.org/cgi/content/full/286/15/1863. Acesso em: 23 jan. 2008. 
WINTER, D. A. Human balance and posture during standing and walking. Gait and Posture, Amsterdam, v. 3, n. 4, p. 193-214, 1995. http://dx.doi.org/10.1016/0966-6362(96)82849g.

WU, W. H.; MEIJER, O. G.; BRUIJN, S. M.; HU, H.; JAAP, H.; van DIEEN, J. H.; LAMOTH, C. J. C.; van ROYEN, B. J.; BEEK, P. J. Gait in Pregnancy-related Pelvic girdle Pain: amplitudes, timing, and coordination of horizontal trunk rotations. Europe Spine Journal, Philadelphia, v. 17, p. 1160-1169, 2008. http://dx.doi.org/10.1007/s00586-008-0703-0.

WU, W.; MEIJER, O. G.; JUTTE, P. C.; UEGAKI, K.; LAMOTH, C. J. C.; WOLF, G. S. de; van DIEEM, J. H.; WUISMAN, P. I. J. M.; KWAKKEL, G.; VRIES J. I. P.; BEEK, P. J. Gait in patients with pregnancy-related pain in the pelvis: an emphasis on the coordination of transverse pelvic and thoracic rotations. Clinical Biomechanics, Oxford, v. 17, n. 9-10, p. 678-686, 2002. http://dx.doi.org/10.1016/S0268-0033(02)00109-2.

WU, W.; MEIJER, O. G.; LAMOTH, C. J. C.; UEGAKI, K.; VAN DIEEN, J. H.; WUISMAN, P. I. J. M.; VRIES, J. I. P.; BEEK, P. J. Gait coordination in pregnancy: transverse pelvic and thoracic rotations and their relative phase.

Clinical Biomechanics, Oxford, v. 19, p. 480488, 2004.

http://dx.doi.org/10.1016/j.clinbiomech.2004.02.00 3.

Endereço:

Luana Mann

Rua Dep. Antônio Edu Vieira, 694, servidão anexa, Bloco B, apto 203. Residencial Estudantil Santa Rita de Cássia. Bairro Pantanal.

Florianópolis SC Brasil 88040-000.

Telefone: (48) 9109.6545

e-mail: luanamann@gmail.com

Recebido em: 6 de novembro de 2009. Aceito em: 28 de abril de 2010. 